\title{
On The Study of Development of X Band Metamaterial Radar Absorber
}

\author{
Mahmoud A. Abdalla ${ }^{1^{*}}$ and Zihrun $\mathrm{Hu}^{2}$ \\ ${ }^{1}$ Electronic Engineering Department, MTC University, Cairo, Egypt, \\ ${ }^{2}$ School of Electrical and Electronic Engineering, University of Manchester UK, \\ *E-mail : maaabdalla@ieee.org
}

\begin{abstract}
A new development of metamaterial applications in radar absorbers for $\mathrm{X}$ band is introduced. Two modifications were suggested based on two different approaches which are a new called fan shaped resonator absorber and a modified high impedance metamaterial absorber. Both approaches introduce thin radar absorber (5.3\% at centre frequency) with wide bandwidth and high absorption level. The theoretical concepts of each design are explained and validated using full wave simulation. Results illustrate that the new development can achieve wider bandwidth, multiple operating bands; the increase in bandwidth is up to 8 times the conventional one. Moreover, the reported absorbers have capability to operate with different polarizations.
\end{abstract}

\section{Introduction}

A great interest has been paid on studying the new characteristics of using artificially constructed structures known as "metamaterials". Metamaterials were introduced for the first time as a theoretical concept in 1967 by the Russian physicist Victor Veselago. He illustrated that if a material can exhibit negative electric permittivity $\varepsilon$ and permeability, it has unique electromagnetic properties [1].

The electromagnetic constitutive parameters of a medium, the electric permittivity $(\varepsilon)$ and magnetic permeability $(\mu)$, are used to describe the material behavior on applying an electromagnetic wave. In 1996, Pendry [2] has realized an artificial $3 \mathrm{D}$ array of thin straight wires that can demonstrate negative permittivity. In 1999, Pendry [3] proposed an artificial magnetic medium consisting of an array of split ring resonators (SRRs) structure which can demonstrate negative permeability. A Composite medium with simultaneous negative permeability and permittivity has been introduced in 2000 [4].

The use of radar absorbing materials (RAM) has been increased in past years in different applications such as communication antennas, anechoic chambers, electromagnetic interference (EMI), electromagnetic compatibility (EMC), and stealth technology. The advance in the use of RAM applications aims to improve their performance in terms of their absorption level, operation bandwidth, enhancement of its physical thickness limitations and their properties for different oblique incident and incident polarizations [5-9].

The design of thin absorbing layers for radar cross section reduction is a challenging task, because the thickness reduction leads to a decrease of the bandwidth. Another problem is the absorber capability for all possible incident polarizations. Metamaterial use in radar absorber has contributed in overcoming the initial problem to introduce very thin metamaterial radar absorber. However, the operating bandwidth and the capability of operation with different polarization is still a challenge.

In this paper, we introduce the novel use of different configurations of metamaterial absorbers for the purpose of enhancement the performance of the RAM. The enhancement includes increasing the absorption level and achieving wide band metamaterial absorber. Comparisons between the performances of the metamaterial absorber based on conventional metamaterial configuration versus our modified ones are introduced. For this purpose, two different RAM were designed at the $\mathrm{X}$ band. The full wave electromagnetic wave simulations were employed to calculate the reflection coefficient for a normal incident plane wave.

The commercial software (Ansoft) was employed for co polarized and cross polarized incident wave. The theoritical explanation of each reported absorber operation is discussed. Results illustrate that both proposed absorbers can achieve wider bandwidth compared to the conventional one. Also, results demonstrated the reported absorber capability of operation of different polarizations.

\section{Fan Shaped SRR RAM}

\subsection{Structure and Theory}

The design of thin absorbing layers for radar cross section reduction is a challenging task, because the thickness reduction leads to a decrease of the bandwidth. The simplest metamaterial absorber structure based on the use of conventional rectangular shaped SRR, shown in Figure 1 (a) 
is mainly narrow band and also it works for only for either co polarized and cross polarized wave.

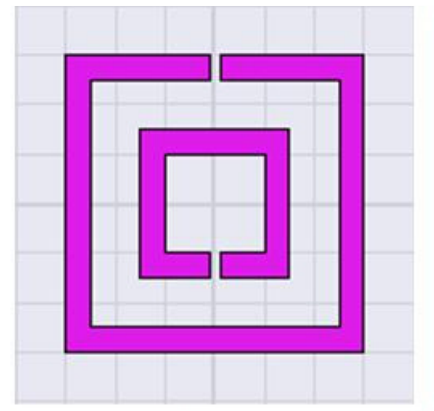

(a)

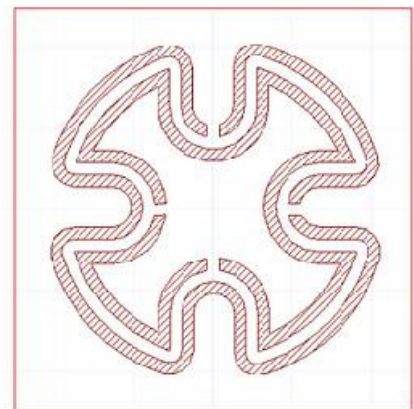

(b)
Figure 1: A unit cell geometry of (a) a rectangular SRR (b) a fan shaped SRR

The equivalent circuit of the metamaterial transmission line RAM is shown in Figure 2 (a) and the SRR equivalent circuit is shown in Figure 2 (b).

By using a periodic array of SRR over a thin lossy substrate, it can satisfy the absorber equivalent circuit shown in Figure 2 (a). In our work, the principles of achieving wide/multi band absorber may be achieved by proposing new resonator geometry, named a fan shaped SRR. The idea of increasing bandwidth of the resonator RAM is based on using SRR geometry that can enhance the parasitic effect so that the whole metamaterial RAM can resonate at different frequencies. Also, the proposed resonator has symmetric gap in all directions which makes it good candidate to operate as a radar absorber for both co polarized and cross polarized incident electromagnetic wave.

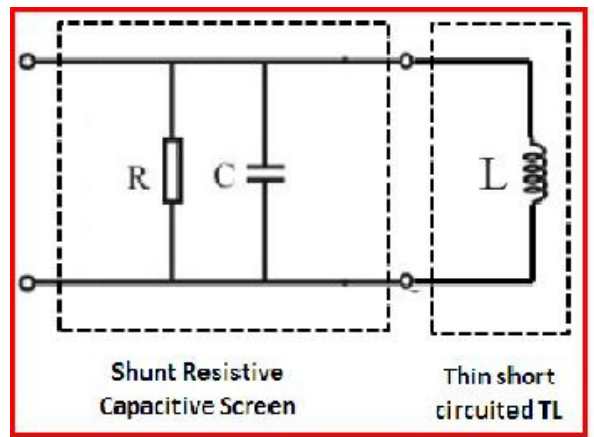

(a)

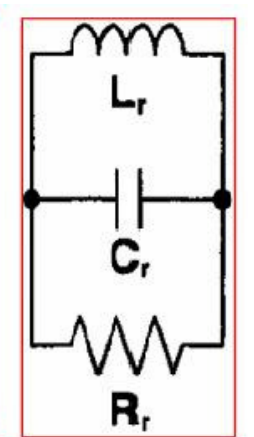

(b)
Figure 2: An equivalent circuit of the metamaterial transmission line RAM. (b) A SRR equivalent circuit.

As a suggestion of solving the above drawbacks, in this work, we suggest the use of new fan shaped SRR shown in Figure 1 (b). For the sake of good comparison, two different RAM layers were designed using conventional SRR and Fan shaped SRR on a lossy FR4 substrate whose relative dielectric constant $\mathrm{gr}=4.4$, a dielectric loss tangent, $\tan \delta=$ 0.02 , and thickness of $1.6 \mathrm{~mm}$. The operating frequency and bandwidth can be designed by the proper selection of the employed unit cell. The fan shaped resonator has an outer radius of $2.15 \mathrm{~mm}$. All the lines thickness and gaps are equal to $0.15 \mathrm{~mm}$.

\subsection{Results}

The performance of the fan shaped resonator RAM is presented in this section. The full wave electromagnetic wave simulations were employed to calculate the reflection coefficient for a normal incident plane wave. The commercial software (Ansoft) was employed for co polarized and cross polarized incident wave.

The simulated reflection coefficient for normal incidence of electromagnetic waves upon the metamaterial absorber implemented using rectangular SRR versus the fan shaped SRR are shown in Fig. 3 for the case of co polarized wave. As illustrated in Figure 3, the conventional rectangular SRR RAM can satisfy electromagnetic wave absorption at approximately $9.5 \mathrm{GHz}$ with close to $-15 \mathrm{~dB}$ reflection coefficient over a narrow bandwidth $(10 \mathrm{~dB}$ fractional bandwidth is only $1 \%$ ). On the other hand, the fan shaped SRR can demonstrate electromagnetic wave absorption centered at the same frequency $9.5 \mathrm{GHz}$ with fractional 10 $\mathrm{dB}$ bandwidth of approximately $4.5 \%$. Moreover, the fan shaped SRR absorber can demonstrate a second absorption band at approximately $11.7 \mathrm{GHz}$ which has better than -20 $\mathrm{dB}$ reflection coefficient over approximately $10 \mathrm{~dB}$ fractional $8 \%$ bandwidth. This means that the fan shaped SRR metamaterial absorber can increase the bandwidth up to eight times compared to conventional SRR absorber.



Figure 3: The simulated reflection coefficient of rectangular SRR and fan shaped SRR metamaterial absorber for copolarized wave.

Another advantage of the fan shaped is it can satisfy the electromagnetic absorption for either co polarized and cross polarized wave. For confirming of the polarization capability, the simulated reflection coefficients of both aforementioned absorbers assuming a cross polarized incident wave are shown in Figure 4. As shown in the figure, the fan shaped metamaterial absorber can demonstrate almost the same absorption for the two possible cases of co polarized and cross polarized electric field incidence. On contrast to fan shaped case, the 
conventional rectangular SRR failed to demonstrate any small reflection coefficient over the frequency band of interest as it is nearly $0 \mathrm{~dB}$.

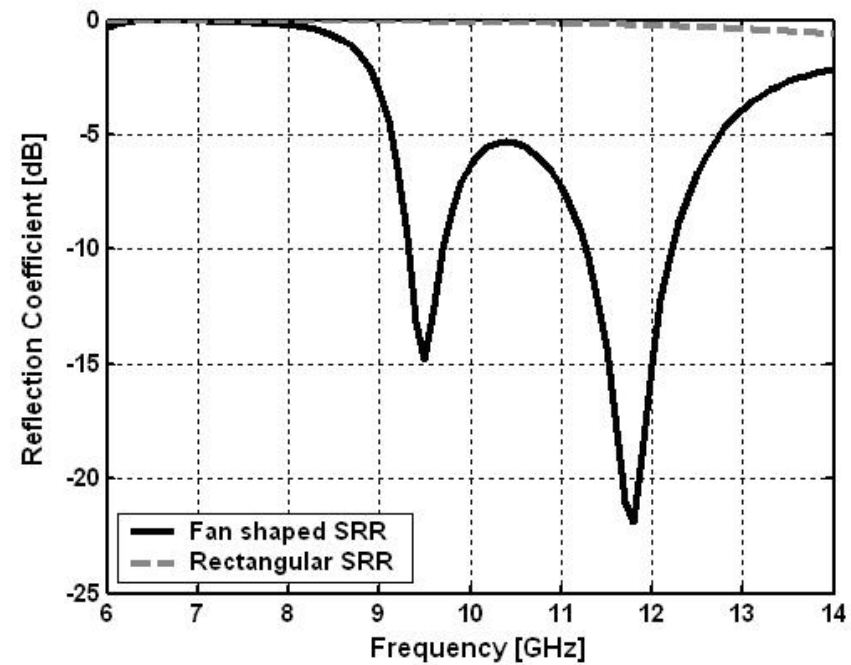

Figure 4: The simulated reflection coefficient of rectangular SRR and fan shaped SRR metamaterial absorber for crosspolarized wave.

\section{Slot High Impedance Metamaterial RAM}

\subsection{Structure and Theory}

Another proposal of metamaterial absorber is based on the use of high impedance surface property that can be achieved using metamaterials. This metamaterial absorber is characterized as a thin absorber. Different approaches for the high impedance thin absorber have been proposed by Engheta [9], Kern and Werner [10], and Gao et el using the Sievenpiper high impedance ground plane (HIGP) [11]. However, it is characterized with narrow bandwidth and low absorption level.

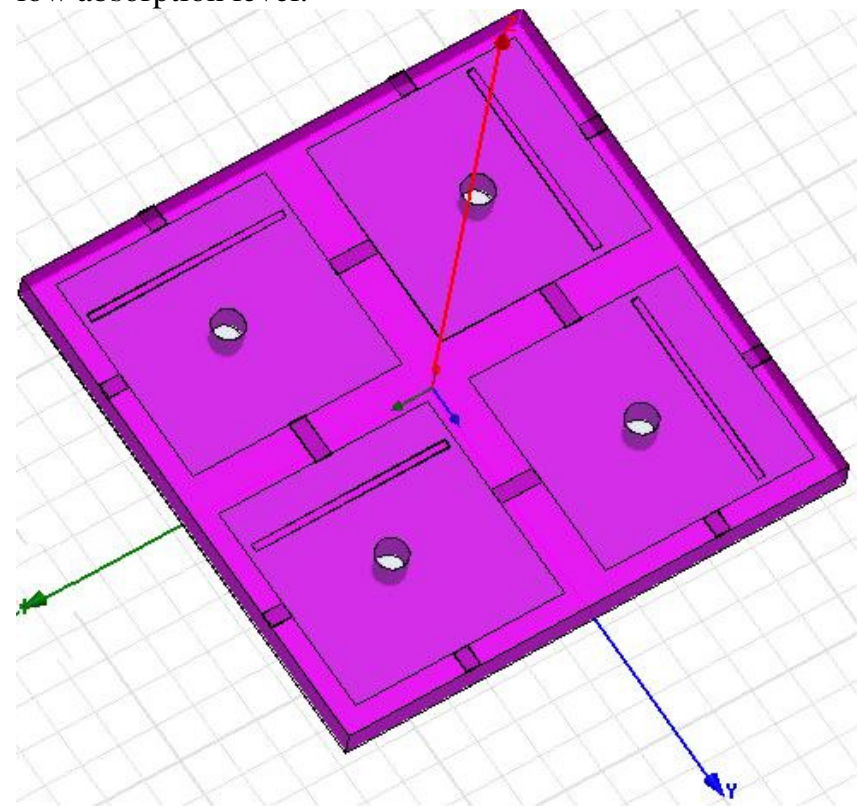

Figure 5: The 3-D sketch of the modified HIGP metamaterial absorber
In this section we introduce a modified high impedance metamaterial absorber to have wider and higher absorption capability compared to conventional high impedance absorber and also capable of operation for both possible cases of polarization. The 3-D sketch of the proposed absorber is shown in Figure 4. As shown in the figure, the proposed absorber is formed using rectangular patches connected to ground through conductor pins. The rectangular patches are printed on the top of an FR4 lossy substrate with relative dielectric constant $\varepsilon_{\mathrm{r}}=4.4$, a dielectric loss tangent, $\tan \delta=0.02$, and thickness of 1.6 $\mathrm{mm}$.

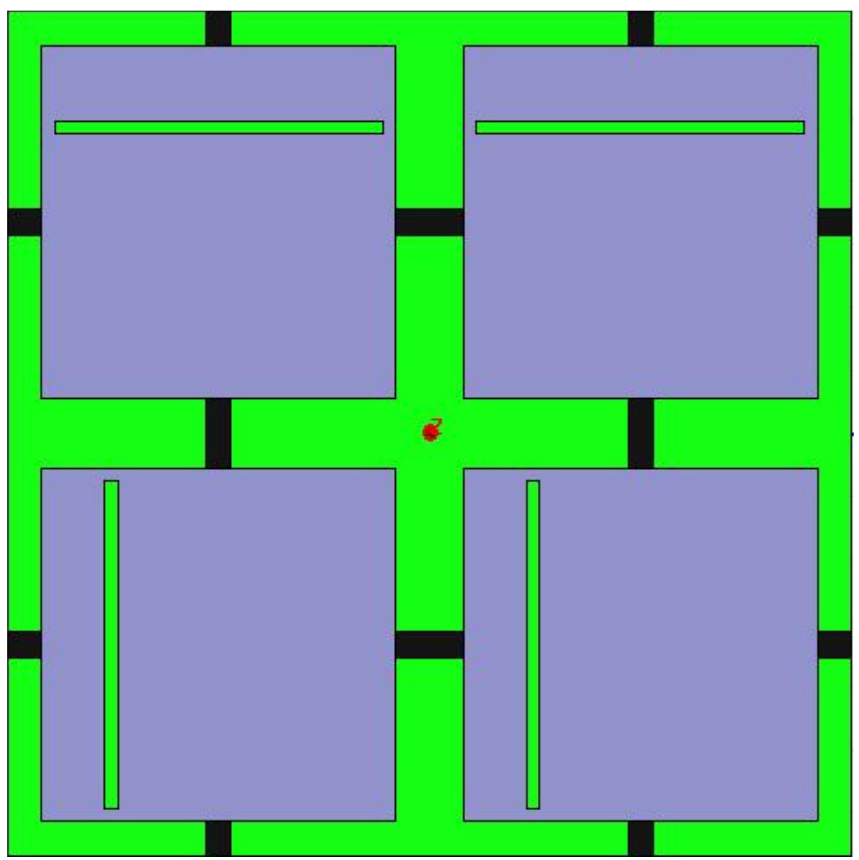

Figure 6: A 2 X 2 unit cell geometry of the modified high impedance metamaterial absorber

The layout of the proposed modified high impedance metamaterial absorber is shown in Figure 6. As shown in the figure, the rectangular patches were modified by cutting rectangular slots in asymmetric configurations. The inserted slots were designed as two horizontal slots for two patches and two vertical slots for the other slots. The reason behind this is to ensure the proposed absorber capability to operate well with both possible electric field polarizations of incident electromagnetic fields. In this case, the $2 \mathrm{X} 2$ asymmetric cells can be considered one unit cell in modeling the reported absorber. All the used slots have the same dimensions $(5.2 \mathrm{~mm}$ X $0.2 \mathrm{~mm}$ ). The employed patches are square shaped whose length is $5.2 \mathrm{~mm}$. Surface mount resistances of $375 \Omega$ are used to connect the patches.

\subsection{Results}

The performance of the proposed modified HIGP metamaterial resonator is presented in this section. The RAM performance was investigated using the electromagnetic full wave electromagnetic wave simulations to calculate the reflection coefficient for a normal incident plane wave. The commercial software (Ansoft) was employed for co polarized and cross polarized 
incident wave. Also, the results are discussed as a comparative study between the modified HIGP metamaterial absorber and the conventional one.

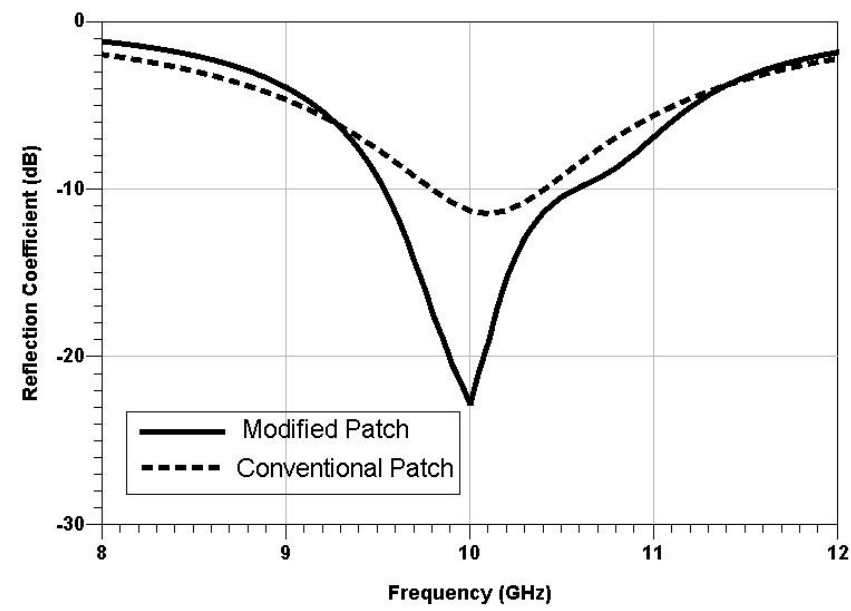

Figure 7: The simulated reflection coefficient of the modified slotted patch RAM for co polarization case.

The simulated reflection coefficient of the modified HIGP patch versus conventional one is shown in Figure 7. As shown in the figure, the modification done to the patch has resulted in increasing the return loss at the centre frequency, $10 \mathrm{GHz}$ to better than $20 \mathrm{~dB}$ instead of approximately 12 $\mathrm{dB}$. The modified patch RAM has a minimum $10 \mathrm{~dB}$ return loss from $9.5 \mathrm{GHz}$ to $10.5 \mathrm{GHz}$ whereas it is only from 9.85 $\mathrm{GHz}$ to $10.4 \mathrm{GHz}$ in case of conventional patch RAM. In other words, in terms of the fractional bandwidth, it is increased to $10 \%$ instead of $5.5 \%$ for conventional HIGP absorber which is almost double value enhancement.

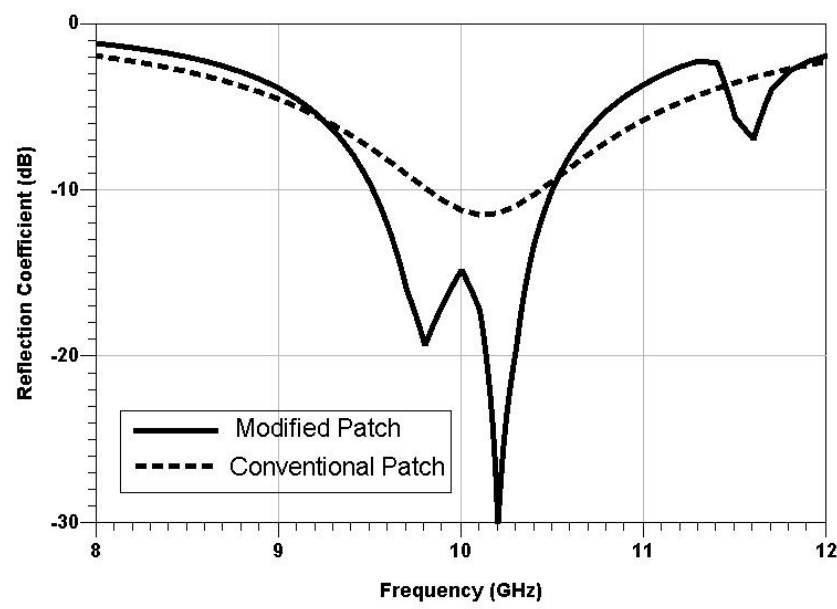

Figure 8: The simulated reflection coefficient of the modified slotted patch RAM for cross polarization case

For the sake of completeness, the modified absorber was reinvestigated for the case of cross polarization incident wave. The study is also done as a comparative study versus the conventional HIGP metamaterial patch antenna and the results is illustrated in Figure 8. As shown in the figure, the conventional patch HIGP metamaterial absorber has similar $10 \mathrm{~dB}$ narrow bandwidth and its reflection coefficient can not go below $-12 \mathrm{~dB}$ within this bandwidth; similar to the co polarized incident case illustrated in Figure 5. On the other hand, the modified patch HIGP metamaterial absorber has wider $10 \mathrm{~dB}$ which is almost from $9.5 \mathrm{GHz}$ to $10.5 \mathrm{GHz}$ which is approximately the same $10 \mathrm{~dB}$ reflection coefficient bandwidth of the modified patch HIGP metamaterial absorber illustrated in Figure 5. However, it is obvious that the modified patch HIGP metamaterial absorber response is different within this bandwidth from the co-polarized incident wave. But, these changes can be accepted since they take places below $-15 \mathrm{~dB}$ at which only it reveal no more that only $3 \%$ of the incident power may reflect back.

\section{Conclusions}

A new development for wide/multi band metamaterial radar absorber material using new type of split ring resonator, called fan shaped SRR and modified HIGP metamaterial absorber has been discussed. The performance of the two reported metamaterial absorbers were investigated and compared versus the conventional rectangular SRR absorber/HIGP patch metamaterial absorber. The results confirm that both reported new developed metamaterial absorbers can introduce wider and multiple bands compared to conventional ones. The bandwidth for fan shaped SRR absorber has dual band with approximately $8 \%$ bandwidth, up to 8 times increase compared with conventional one. The modified HIGP absorber has almost $10 \%$ bandwidth which is almost doubled the conventional one. Also, both absorber absorbers can sustain their performance for both co polarized and cross polarized waves.

\section{References}

[1] Veselago V. G. "The electrodynamics of substances with simultaneously negative values of \&epsiv; and \&mu," Soviet Physics - Uspekhi, vol. 10, 509-14, 1968.

[2] Pendry J. B., A. J. Holden, D. J. Robbins, and W. J. Stewart, "Low frequency plasmons in thin-wire structures," Journal of Physics: Condensed Matter, vol. 10, 4785-809, 1998.

[3] Pendry J. B., A. J. Holden, D. J. Robbins, and W. J. Stewart, "Magnetism from conductors and enhanced nonlinear phenomena," IEEE Transactions on Microwave Theory and Techniques, vol. 47, 2075-84, 1999.

[4] Smith D. R., W. J. Padilla, D. C. Vier, S. C. NematNasser, and S. Schultz, "Composite medium with simultaneously negative permeability and permittivity," Physical Review Letters, vol. 84, 4184-7, 2000

[5] Amano M. and Y.Kotsuka, "A novel microwave absorber with surfaceprinted conductive line patterns," in IEEE MTT-S Digest, Jun. 2002,1193-1196.

[6] Costa F., A. Monorchio, and G. Manara, "Ultra-thin absorber by using high impedance surfaces with frequency selective surfaces," in Proc. IEEE Int. Symp. on Antennas Propagation., Honolulu, USA, 2007,.861864. 
[7] A. Tennant and B. Chambers, "A single-layer tunable microwave absorber using an active FSS," IEEE Microw. Wireless Compon. Lett., Vol. 14, No. 1, 4647, 2004.

[8] Shen Z. and H Wang "On the optimum design of a thin absorbing screen", in Proc, Antennas and Propagation Society International Symposium, Honolulu, USA 2007 $6039-6042$.

[9] Engheta, N.: 'Thin absorbing screens using metamaterial surfaces'. IEEE Antennas and Propagation Society (AP-S) Int. Symp. and USNC=URSI National Radio Science Meeting, San Antonio, TX, USA, 2002, pp. 16-21

[10] Kern, D.J., and Werner, D.H.: 'A genetic algorithm approach to the design of ultra-thin electromagnetic bandgap absorbers', Microw. Opt. Technol. Lett., 2003, 38, (1), pp. 61-64

[11] Q. Gao, Y. Yin, D.-B. Yan and N.-C. Yuan, "Application of metamaterials to ultra-thin radarabsorbing material design", Electronics Lett., 2005, Vol. 41, No. 17, pp 17-18. 